\title{
Massively parallel calculations of the electronic structure of non-periodic micro-crystallites of transition metal oxides
}

\author{
Marek T. Michalewicz \\ Division of Information Technology, Commonwealth Scientific and Industrial Research Organization, 723 Swanston Street, \\ Carlton, Victoria 3053, Australia
}

Received 9 September 1993

\begin{abstract}
We report massively parallel computations of the electronic density of states of a non-periodic micro-crystallite of rutile $\mathrm{TiO}_{2}$ with a sample of 491520 atoms. Mathematically, the problem is equivalent to solving an $n \times n$ eigenvalue problem, where $n \sim 2500000$. We used MasPar MP-1 and MasPar MP-2 autonomous SIMD computers with up to 16384 processing elements (PEs) with the entire computing time $\sim 1.5 \mathrm{~h}$ (on $4 \mathrm{~K}$ MasPar MP-2). We used the equation of motion method and the non-trivial tight binding model in our calculations and conclude that these methods when implemented on the massively parallel SIMD computer architectures, are very well suited for studying the electronic structure of complex non-periodic systems with both point and extended defects.
\end{abstract}

\section{Introduction}

The purpose of this paper is to report on an implementation of the equation of motion method for the computation of the electronic structure of non-periodic, disordered transition metal oxides on massively parallel SIMD machines. The equation of motion method [1,2] and the algorithm used for the calculations is well known [3,4]. The CRAY vector implementation of this method has been communicated by the present author and co-workers recently [5]. The program EQ_Of_MOTION has been catalogued in this journal (catalog number: ACJD).

The most challenging aspect of the current project was to adapt the algorithm [5], originally devised for vector processors for the massively parallel SIMD computer. By performing the computations on the massively parallel machine with very large number of processors we were able to increase the sample size over a hundredfold with respect to what was previously done on the CRAY supercomputer. We can now easily model complicated extended surface defects, such as atomic steps, shear defects, islands, etc. The sample can be both periodic or non-periodic. The method could also be easily adapted to study different crystallographic planes bounding the micro-crystallites of nearly macroscopic sizes. Effectively, we are now in a position to model the sample shape in a stone cutter fashion. The importance of such studies is obvious in the context of fabricated defects, superstructures, passivation layer properties, corrosion, surface diffusion in the presence of extended surface defects and many others. It might lead also to studies of localization in the limit of a macroscopically large samples.

Correspondence to: M.T. Michalewicz, Division of Information Technology, Commonwealth Scientific and Industrial Research Organization, 723 Swanston Street, Carlton, Victoria 3053, Australia.

E-mail: marek@mel.dit.csiro.au. 
We ran the program to compute the total and local electronic densities of states for the $\mathrm{TiO}_{2}$ samples with sizes from the few unit cells up to $41.5 \times 41.5 \times 1.5 \mathrm{~nm}$, the largest sample consisting of 491520 atoms or 81920 unit cells. The program is fully scalable in the number of atoms, i.e. the time required to run it is independent of the size of the system in $x$ and $y$ directions (PE mesh) ${ }^{\# 1}$ and is linear in $z$ direction (memory axis) [6]. The total CPU time for the largest sample, on MasPar2 computer with 16384 processing elements (PEs) is estimated to be $\sim 1.5 \mathrm{~h} \# 2$. The program was written in FORTRAN90 (the performance was increased by using MasPar MP FORTRAN compiler parallel performance directives).

The results of our computations, including the results for electronic surface density of states (DOS) on the surfaces with atomic steps will be communicated elsewhere [7].

Here we would like to describe the methods of mapping a non-trivial tight binding Hamiltonian model for the rutile structure [8] onto the two-dimensional mesh of processing elements in a way which optimally utilize the local memory on each PE and the communication between the neighbouring PEs.

There is a number of reasons why we chose $\mathrm{TiO}_{2}$ for our study. First, $\mathrm{TiO}_{2}$ is of technological importance in the areas of energy conversion, corrosion inhibition, fiber optic coatings, photolysis of water [9] catalysis [10] and electronic materials. Secondly, $\mathrm{TiO}_{2}$ and its surfaces as well as the surface point and extended defects, have been intensively studied experimentally [11-14]. The effect of the surface defects on the adsorption and desorption of small molecules on $\mathrm{TiO}_{2}$ surfaces has been reported in refs. [15-18]. Lastly, the rutile structure of $\mathrm{TiO}_{2}$ poses a very interesting problem when one attempts to map it onto $2 \mathrm{D}$ mesh of processors. We will discuss this in detail in the following sections.

In recent years the equation of motion method was used to study the transition metal oxide, $\mathrm{TiO}_{2}$, with large concentration of oxygen vacancies (up to 10\%) and for $2 \mathrm{D}[3,4]$ and $3 \mathrm{D}$ [4] samples with up to $3840 \mathrm{Ti}$ and $\mathrm{O}$ atoms. The computed properties included total and local density of states [4], surface density of states [19] and conductivity [20]. The electronic structure of $\mathrm{TiO}_{2}$ surfaces, both ideal and with point defects was also studied theoretically using the Green's function, scattering-theoretic method [21].

The equation of motion method was also adapted for the evaluation of other electronic properties (mainly of amorphous silicon), such as: electronic conductivity via Kubo formula [22], localization [23], spectral functions [24], interband linear optical properties [25], electronic structure and conductivity [26], the Hall coefficient [27] or the electronic structure of hydrogenated a-Si [28].

The organization of the paper is the following. In section 2 we state the computational problem, briefly outlining the equation of motion method. In section 3 we discuss the mapping of the rutile structure onto a 2D mesh of processing elements and the communication between the PEs. In the final section we discuss the directions for the further developments of this massively parallel method.

\section{Computational problem}

The equation of motion method provides an efficient way to compute the electronic and vibrational densities of states of disordered solids [1,2]. Condensed matter physicists are perhaps more familiar with the recursion method $[29,30]$. The discussion of the relative merits and a comparison of the two methods is outside the scope of this work and can be found in refs. [31,32,3].

The equation of motion method is intuitively very simple. All computations are performed in the direct space, so that, with some experience one can visualise all the computational and spatial relationships in any particular crystallographic structure and map it onto the computational mesh of

\footnotetext{
\#1 This is true as long as the size of system in the $x-y$ plane does not exceed the size of the PE mesh. Currently the limit on MasPar is $128 \times 128$.

\#2 The program was run on MasPar MP-1 (1K, 4K, 16K PEs) and MasPar MP-2 (1K, 4K PEs). The 16K result is estimate from nearly ideal scaling. The time on $4 \mathrm{~K}$ MasPar MP-2 was $1 \mathrm{~h} 24 \mathrm{~min}$.
} 
processors. The method effectively computes all the eigenvalues of a square matrix (Schrödinger equation) without resorting to a direct diagonalization and puts no stringent restriction on the range of impurity potential [33]. The equation of motion method for the system with many orbitals per site was described in detail in refs. [4,5]. We refer to these papers and here quote only the most crucial steps.

The time dependent Schrödinger equation

$$
\mathrm{i} \hbar \partial \Psi / \partial t=H \Psi
$$

is solved and the result is Fourier transformed in time to obtain the quantities such as the density of states which are of interest at the end of the calculation. The tight binding Hamiltonian is written in the form

$$
H=\sum_{i, \mu} \epsilon_{i, \mu} c_{i, \mu}^{\dagger} c_{i, \mu}+\sum_{i, \mu ; j, \nu}\left(t_{i, \mu ; j, \nu} c_{i, \mu}^{\dagger} c_{j, \nu}+\text { h.c. }\right),
$$

where the $i$ are site indices, the Greek letters $\mu, \nu$ are indices labelling orbitals and the sum on (ij) is over neighbours on the lattice. We define a Green function

$$
G_{i, \mu ; j, \nu}(t)=-i \Theta(t)\left\langle\left\{c_{i, \mu}(t), c_{j, \nu}^{\dagger}(0)\right\}\right\rangle
$$

and an amplitude

$$
F_{i, \mu}(t)=\sum_{j, \nu} a_{j, \nu} G_{i, \mu ; j, \nu}(t)
$$

The coefficients $a_{j, \nu}$ can be chosen in various ways depending on what quantity is of particular interest $[4,5]$. The equation of motion for $F_{i, \mu}$ is

$$
\mathrm{i} \hbar \partial F_{i, \mu} / \partial t=\sum_{j, \nu} H_{i, \mu ; j, \nu} F_{j, \nu} .
$$

The equation of motion is integrated using the formal solution

$$
\boldsymbol{F}\left(t_{l+1}\right)=\exp (-\mathrm{i} \boldsymbol{H} \delta t) \boldsymbol{F}\left(t_{l}\right),
$$

with the Chebyshev polynomial fit of the exponential function expansion. The computation of the time evolution expressed above is the most time consuming, taking nearly $88 \%$ of the total CPU time of the vector version of the program (ACJD). The fragment of the FORTRAN77 listing where the interactions of the off-diagonal terms of the tight-binding Hamiltonian are summed reads

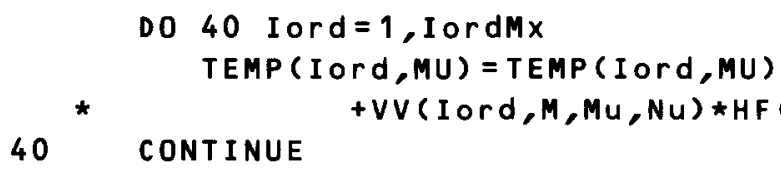

where NVIJK( Iord,M) is the look-up table identifying all the neighbours, with atomic orbital NU, of the Iord atom with the atomic orbital M. As noted in ref. [5], this introduced a restriction on the vectorization of the program, although on the CRAY computers it could be vectorized using the vectorizing gather-scatter operations. On the other hand, on the massively parallel computers, such a look-up table is completely unnecessary. It is the local environment on each particular processing element that defines the physical neighbourhood of each atom. In programming terms, instead of look-up tables we use appropriately defined CSHIFT operations in FORTRAN90. It should be kept in mind when using parallelizing translators such as VAST-90 that look-up tables will be translated literally. 
At the moment it still requires human intervention to impose spatial relations corresponding to a crystallographic system onto the computational mesh.

\section{Parallel implementation}

The physical systems, such as transition metal oxides, described well by the tight binding Hamiltonian with nearest and perhaps second-nearest neighbour interactions render themselves ideally to the topology of the massively parallel computer architecture. The MasPar computer used in the present study is an autonomous single instruction multiple data stream (ASIMD) machine with large number of processing elements (PEs) arranged in the two-dimensional array [6,34]. The communication between the PEs is achieved through the X-Net mechanism, which provides a very fast connections in the so-called NEWS configuration (north-east-west-south). The $x$ and $y$ crystallographic directions coincide with the $x$ and $y$ directions on the PE array. The local memory at each PE provides the third $z$-dimension.

Rutile structure of $\mathrm{TiO}_{2}$ poses some interesting problems for the mapping of atoms on the PE array. It would be a trivial exercise to map a simple cubic structure with nearest-neighbour interactions only. However, the model of the rutile tight-binding Hamiltonian we use in our study, originally proposed by Vos [8], can be called non-trivial. It is characterized by the following features: (i) the sample is three-dimensional; (ii) the rutile structure consists of a tetragonal unit cell with two $\mathrm{Ti}$ and four $\mathrm{O}$ atoms. The titanium atoms occupy the positions $(0,0,0)$ and $\left(\frac{1}{2}, \frac{1}{2}, \frac{1}{2}\right)$ whereas oxygens are at the positions $\pm(x, x, 0)$, and $\pm\left(\frac{1}{2}+x, \frac{1}{2}-x, \frac{1}{2}\right)$, where $x=0.306 \pm 0.001$ [35]. Each Ti cation forms a "dumbell" shape with two neighbouring $\mathrm{O}$ anions. The "dumbell" attached to the $\mathrm{Ti}$ in the centre of the unit cell is rotated by $90^{\circ}$ with respect to the eight "dumbells" attached to the $\mathrm{Ti}$ atoms at the corners of the unit cell. This rotation complicates the mapping somewhat; (iii) there are up to five atomic orbitals at each atom, i.e. five $3 \mathrm{~d}$ orbitals for each $\mathrm{Ti}$ and one $2 \mathrm{~s}$ and three $2 \mathrm{p}$ orbitals for each $\mathrm{O}$ atom; (iv) we include the nearest and the second-nearest neighbours for each atom.

Figure 1 represents a slice of rutile as seen at slight angle towards the (001) plane. If this structure is viewed precisely from the [001] direction then will resemble the mesh of points such as those depicted in fig. 2 . If it is further rotated with respect to the [001] axis by $90^{\circ}$ it will form a mesh similar to what is represented in fig. 3.

Figure 3 depicts the PE array and the projection of rutile in the (001) plane on this array. Each Ti atom (square) is centered on one PE, and the two oxygen atoms forming a "dumbell" with Ti occupy the same processor. Hence there are three atoms on each PE, for each unit cell plane. The black Ti sites are in the $z=0$ plane, and the empty ones are in the $z=0.5$ plane, but they occupy the same memory layer. By doing this we can optimally use the memory on each PE. This projection will naturally form two interlacing, alterating computational sub-meshes which means that all the computations on each sub-mesh are identical. The memory gives the "depth", or the third $z$-dimension of the system. The 1-1 correspondence between the crystallographic rutile system and the PE array can be established in other ways as well. If, for example, we used one PE for one atom (O or Ti) (see fig. 2b in ref. [3]), the communication cost would be much greater, and also we would have to have $\frac{1}{9}$ of the processors empty.

Fig. 1. $\mathrm{TiO}_{2}$ rutile structure. The large balls represent $\mathrm{Ti}$ atoms (green-the top layer, purple-layers below); $\mathrm{O}$ atoms are represented by small red balls. If this structure is slightly rotated according to the right hand rule about the SE-NW diagonal axis, it will collapse into a mesh represented in fig. 2 .

Fig. 2. The (001) surface of the rutile $\mathrm{TiO}_{2}$. The notation is the same as in the previous figure. The green and purple $\mathrm{Ti}$ atoms define two interlacing square sub-lattices. 


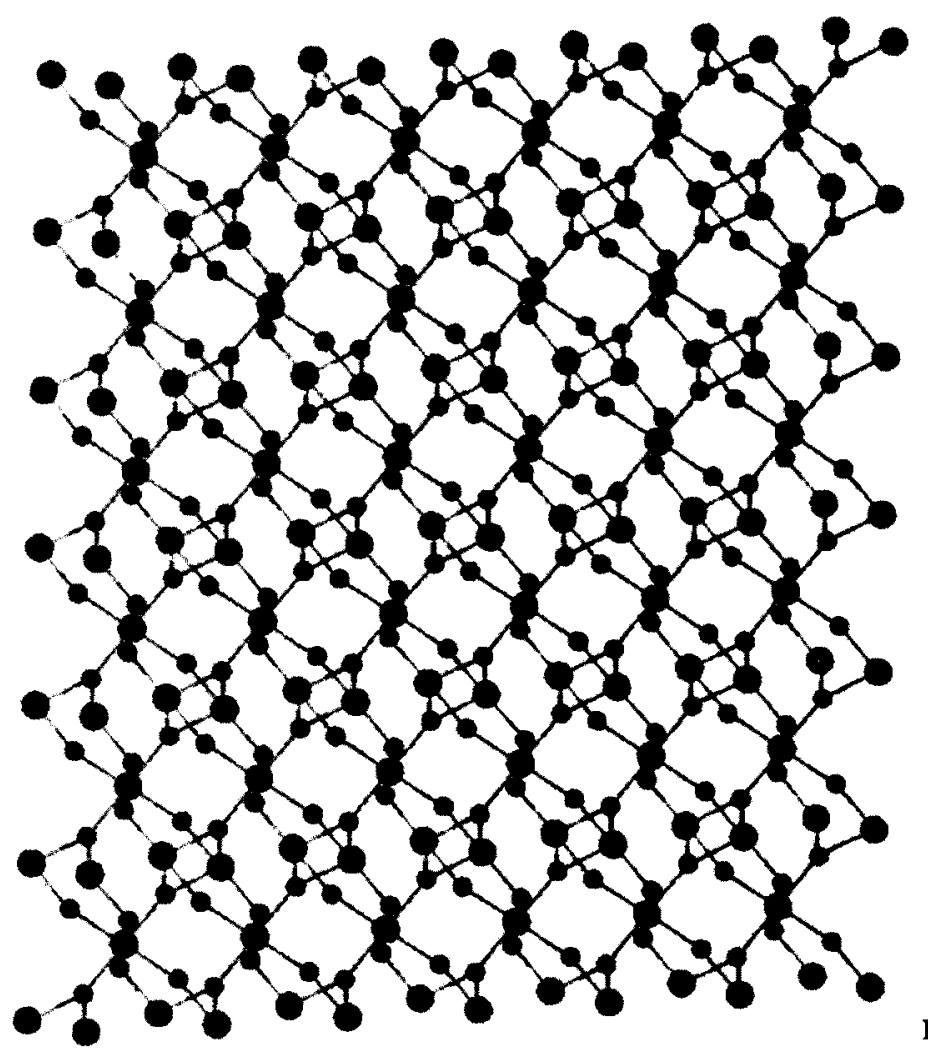

Fig. 1

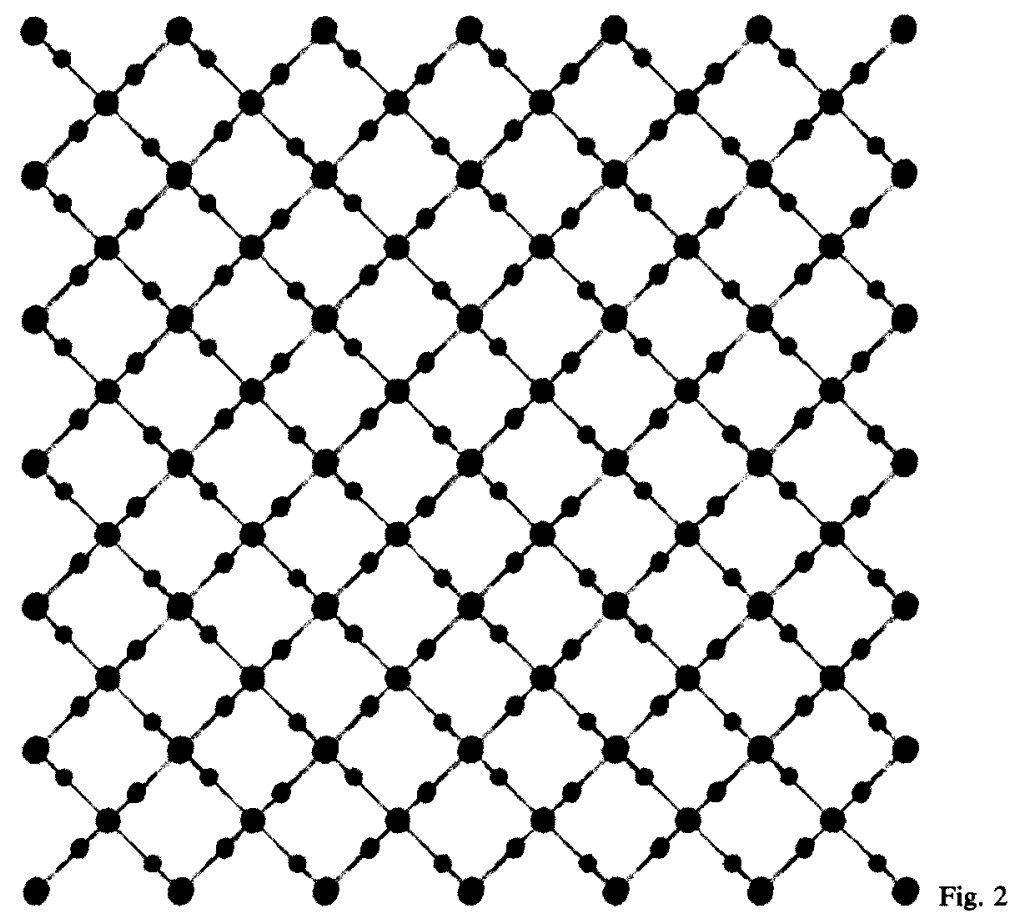




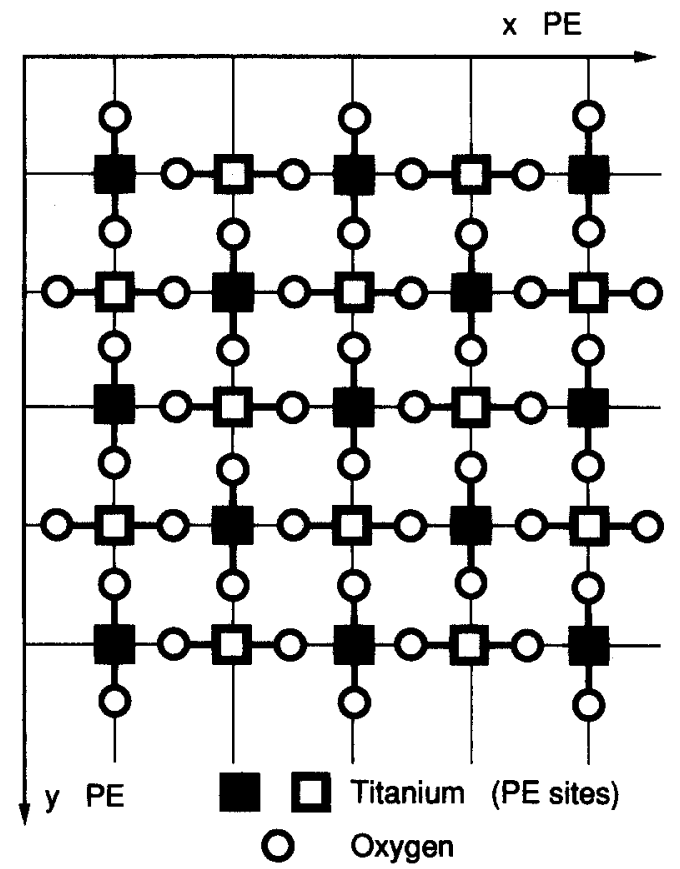

Fig. 3. The (001) surface of the rutile $\mathrm{TiO}_{2}$ mapped on the square mesh of MasPar processors. Each square (Ti atom) corresponds to one processing element. Each pair of $O$ atoms, joined by a solid line, occupies the same PE. The open squares correspond to the purple balls in the previous figure, the solid squares to the green ones.

It is also possible to map the whole unit cell on one PE provided more local memory is available. If we placed six atoms on one PE we would have to reduce the thickness of our sample by half.

The atoms with which each $\mathrm{Ti}$ atom interacts (communicates) are shown in fig. 4 (for $z=0 \mathrm{Ti}$ site). Figure 5 depicts how this local environment was mapped on the processing element mesh. There are 16 neighbours, all in the nearest neighbour position (on the PE mesh). The bold face numbers numerate the neighbours which are in the different memory layers (below). For example, numbers 1 and 2 correspond to the $\mathrm{Ti}$ atoms above and below. They will occupy the same processing element, but have different memory $(z)$ location. Similarily, $O$ atoms 13 and 14 will be on the same PE. The other locations can be deduced by inspecting figs. 4 and 5 .

Figure 6 shows the neighbourhood of $\mathrm{O} 1$ atom in $z=0$. The $\mathrm{O} 2$ atom, located on the same PE will have the neighbourhood like this one, but rotated by $180^{\circ}$ with respect to the normal to the plane of the figure ([001]). Figure 7 represents the mapping of O1 neighbourhood onto PE mesh. Again, by comparing figs. 6 and 7 one can deduce all the communication paths for $\mathrm{O} 1$ atom in $z=0$ memory plane. (The oxygens 10,11, 12 and 13 are not depicted in fig. 6.)

The coding of the interactions of the off-diagonal terms in the tight-binding Hamiltonian will now be written in FORTRAN90 in the following form (for the Ti atom at $z=0$ sub-mesh)

Fig. 4. The local environment of the neighbours interacting with the Ti atom (blue ball). The red balls represent $\mathrm{O}$ atoms, the green ones $\mathrm{Ti}$ atoms.

Fig. 6. The local environment of the neighbours interacting with the $O$ atom (purple ball). The red balls represent $O$ atoms, the green ones $\mathrm{Ti}$ atoms. 

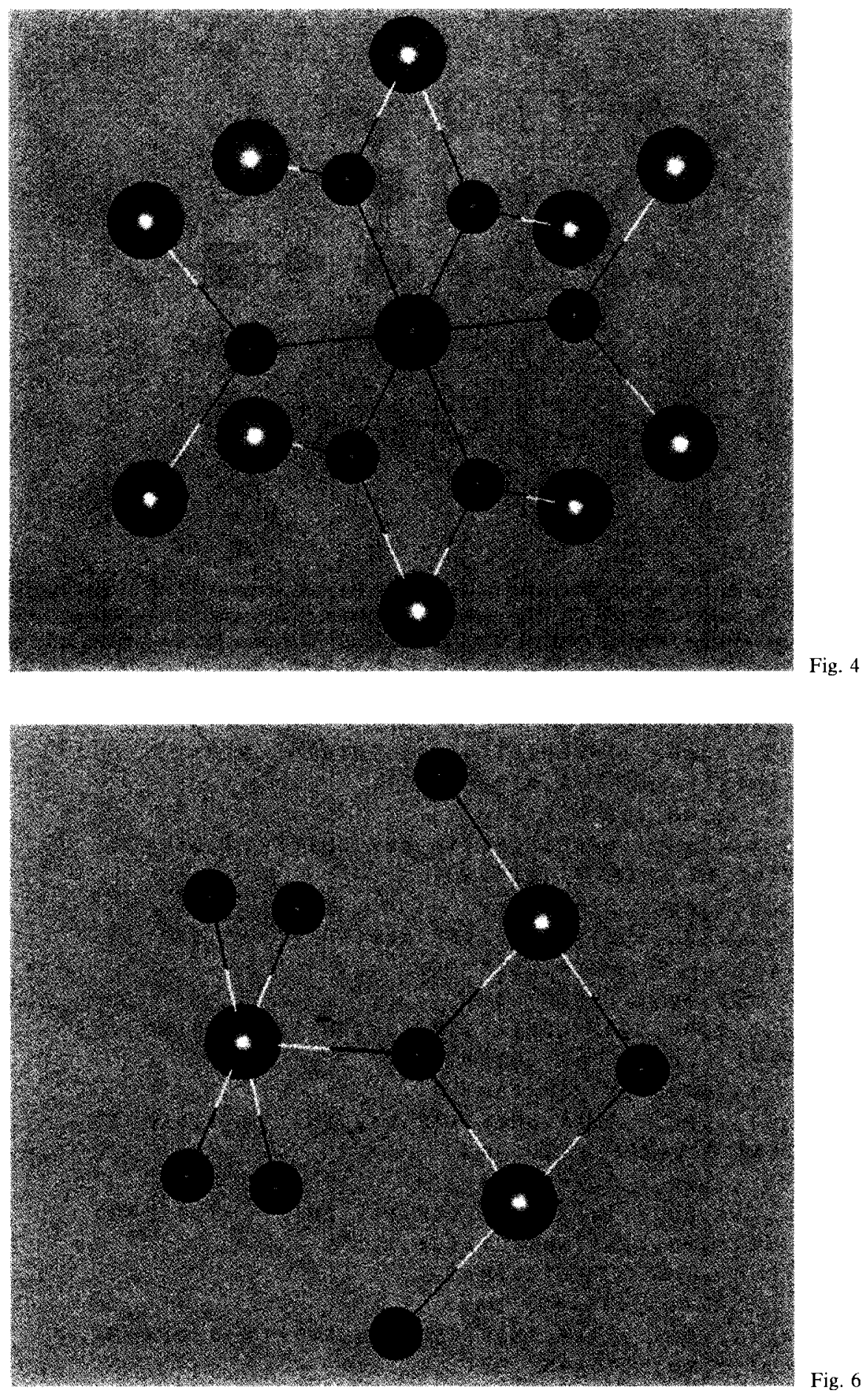


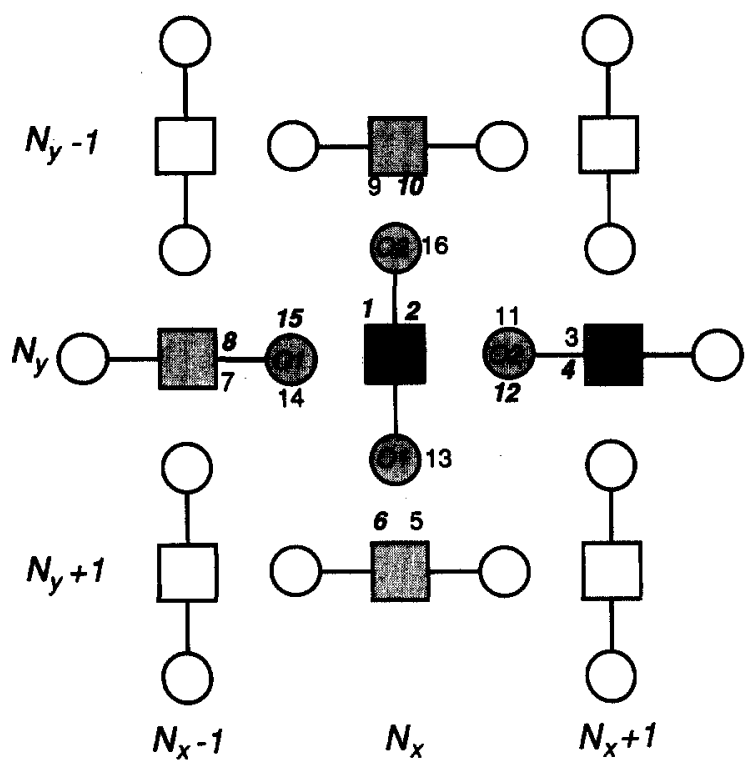

Fig. 5. The mapping of the interacting neighbourhood of the Ti atom (shaded squares (Ti) and circles (O)). There are two types of oxygen atoms on each PE, marked $\mathrm{O} 1$ and $\mathrm{O} 2$. The numbers correspond to the order of neighbours in the program. Italic font corresponds to the higher or lower locations in memory, normal font means the data is at the same memory level.

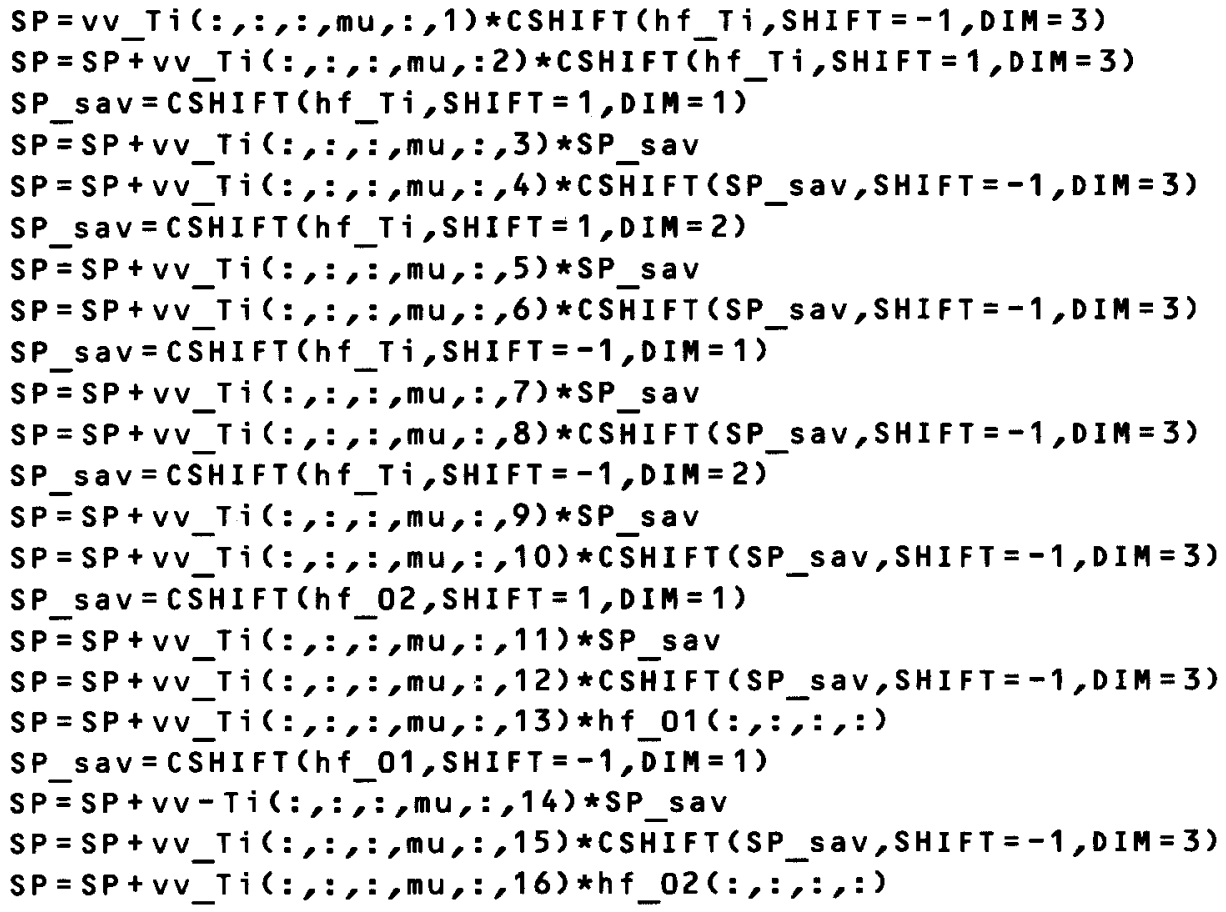

Here we introduced the temporary array SP_sav, since we cannot apply CSHIFT operation twice consecutively on the same array. This also saves some local memory. The first three dimensions in 


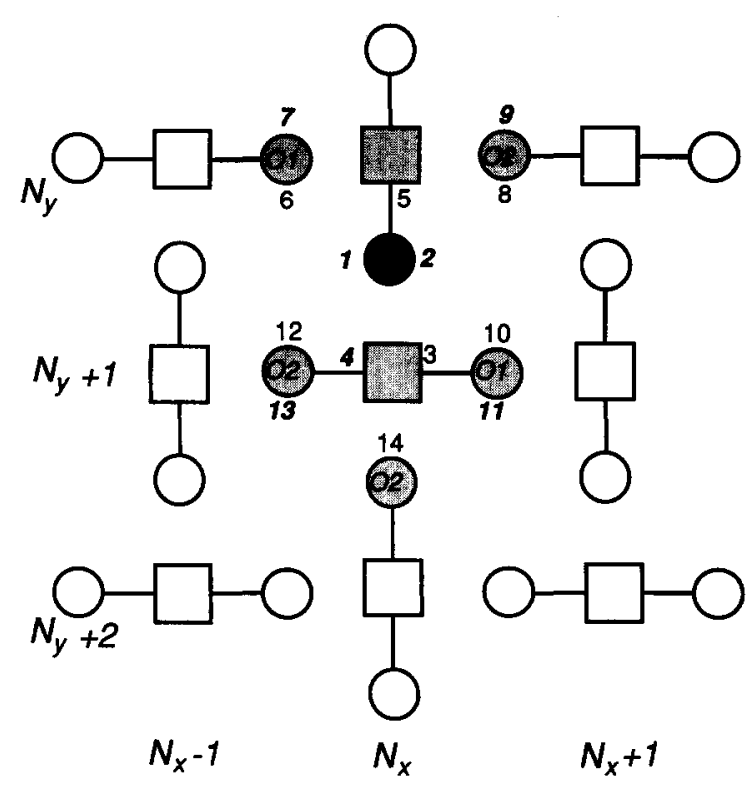

Fig. 7. The mapping of the interacting neighbourhood of the $O$ atom (shaded squares $T i$ and circles $O$ ). The notation convention is the same as in fig 5 .

$v v \_T i(:,:,:, m u,:, n)$ correspond to $x, y$, and $z$ dimensions, the fourth $(\mu)$ and fifth $(\nu)$ correspond to atomic orbitals on the local-atom and the neighbour, respectively. The last dimension corresponds to the neighbour label, as depicted in figs. 4 and 5. This fragment of the FORTRAN90 code can be compared with FORTRAN77 fragment given in the previous section. (It should be realised that the assignment of the neighbour list in FORTRAN77 code required a separate subroutine [5].) Similar coding of the interacting neighbours had to be done for the remaining five atoms in the unit cell.

\section{Conclusions}

The principal result from our study is that by using the massively parallel MasPar computer we were able to study very large samples of $\mathrm{TiO}_{2}$ rutile with up to 491520 atoms. The total CPU time was $\sim 1.5 \mathrm{~h}$ (on MasPar MP-2). The program is fully scalable in the number of atoms. We used nearly all of the distributed memory of 1 Gigabyte on 16K processor MasPar computer but in the future, with the larger local memory machines, it will be possible to study even larger samples of insulating materials or large gap semiconductors using the methods described in this paper. We expect to learn new and interesting physics of non-periodic, disordered solids by studying them on the massively parallel computers and thus obtain results for model systems which will be more similar to experimental samples.

One of the interesting technical problems in applying massively parallel processors to electronic structure studies would be development of the language whose syntax employs the basic space groups defining crystals. Such a language would facilitate automatic generation and optimal projection of arbitrary crystallographic structures on the topology of processing elements network.

With the program described in this report we are able to compute the electronic properties for the samples with cleaved surfaces of different Miller indices, modelling the shape of the microcrystallite in a stone-cutter fashion. The extended surface defects and fabricated features such as stepped surfaces, superlattices, modulated superlattices or doped samples can now be investigated by methods described 
here. All these features can be programmed as software masks which switch-off atoms (PEs) outside the bounds of a sample.

The performance indicators of our program, such as the degree of parallelism (parallel efficiency), the speed of processing and others will be communicated and discussed in detail elsewhere [6].

\section{Acknowledgements}

I wish to thank Dr. I.D. Mathieson, P.G. Whiting, S. McClenahan and S. Pickering for discussions and hints on parallel programming. I gratefully acknowledge collaboration of Mark Priebatsch of MasPar Computer Corporation, Sydney, in running the program on a number of MasPar computers. I thank Dr. C.H.J. Johnson for critical reading of the manuscript and constructive comments. The program described in this paper was developed on the MasPar MP-1 computer at the Ormond Supercomputer Facility which is a joint facility of The University of Melbourne and The Royal Melbourne Institute of Technology. The CRAY part of this project was computed on the CSIRO Supercomputing Facility CRAY Y-MP in Melbourne. I wish to thank Dr. Anneliese Palmer of Biosym Technologies, Inc., Sydney, for her help in producing colour figs. 1, 2, 4 and 6 using Biosym Technologies' Solids Builder and Solids Adjuster.

\section{References}

[1] R. Alben, M. Blume, H. Krakauer and L. Schwartz, Phys. Rev. B 12 (1975) 4090.

[2] D. Beeman and R. Alben, Adv. Phys. 26 (1977) 339.

[3] J.W. Halley and H. Shore, Phys. Rev. B 36 (1987) 6640.

[4] J.W. Halley, M.T. Michalewicz and N. Tit, Phys. Rev. B 41 (1990) 10165.

[5] M.T. Michalewicz, H. Shore, N. Tit and J.W. Halley, Comput. Phys. Commun. 71 (1992) 222.

[6] M.T. Michalewicz and M. Priebatsch, benchmark results, to be published.

[7] M.T. Michalewicz, to be published.

[8] K. Vos, J. Phys. C 10 (1977) 3917.

[9] A. Fujishima and K. Honda, Nature 238 (1972) 37.

[10] Z. Zhang and V.E. Henrich, Surf. Sci. 277 (1992) 263.

[11] V.E. Henrich, Progr. Surf. Sci. 14 (1983) 175; Rep. Prog. Phys. 48 (1985) 1481.

[12] L.A. Bursill and D.J. Smith, Nature 309 (1984) 319.

[13] G.S. Rohrer, V.E. Henrich and D.A. Bonnell, Science 250 (1990) 1239.

[14] G.S. Rohrer, V.E. Henrich and D.A. Bonnell, Surf. Sci. 278 (1992) 146.

[15] K.E. Smith and V.E. Henrich, Surf. Sci. 217 (1989) 445.

[16] E.L. Román, J.L. de Segovia, R.L. Kurtz, R. Stockbauer and T.E. Madey, Surf. Sci. 273 (1992) 40.

[17] U. Diebold and T.E. Madey, J. Vac. Sci. Technol. A 10 (1992) 2327.

[18] J.-M. Pan, B.L. Maschhoff, U. Diebold and T.E. Madey, J. Vac. Sci. Technol. A 10 (1992) 2470.

[19] N. Tit, J.W. Halley and M.T. Michalewicz, Surf. Interface Anal. 18 (1991) 87.

[20] J.W. Halley, Kozlowski, M.T. Michalewicz, W. Smyrl and N. Tit, Surf. Sci. 256 (1991) 397.

[21] S. Munnix and M. Schmeits, Phys. Rev. B 30, (1984) 2202; 31 (1985) 3369; 33 (1986) 4136.

[22] B. Kramer and D. Weaire, J. Phys. C 11 (1978) L5.

[23] D. Weaire and A.R. Williams, J. Phys. C 10 (1977) 1239.

[24] B.J. Hickey and G.J. Morgan, J. Phys. C 19 (1986) 6195.

[25] D. Weaire, B.J. Hickey and G.J. Morgan, J. Phys. Condens. Matter 3 (1991) 9575.

[26] J.M. Holender and G.J. Morgan, J. Phys. Condens. Matter 4 (1992) 4473.

[27] J.M. Holender and G.J. Morgan, Phil. Mag. Lett. 65 (1992) 225.

[28] J.M. Holender, G.J. Morgan and R. Jones, Phys. Rev. B 47 (1993) 3991.

[29] R. Haydock, V. Heine and M.J. Kelly, J. Phys. C 5 (1972) 2845

[30] D.G. Pettifor and D.L. Weaire, eds. The Recursion Method and Its Applications (Springer, Berlin, 1984), and references therein. 
[31] A. MacKinnon, in: The Recursion Method and Its Applications, eds. D.G. Pettifor and D.L. Weaire (Springer, Berlin, 1984) p. 84.

[32] D.L. Weaire and E.P. O'Reilly, J. Phys. C 18 (1985) 1401.

[33] Nacir Tit and J.W. Halley, Phys. Rev. B 45 (1992) 5887.

[34] J.C. Kalb, presented at the 4th Australian Supercomputer Conference, Gold Coast, 1991 and MasPar System Overview, Doc. Par No. 9300-0100, MasPar Corporation.

[35] F. A. Grant, Rev. Mod. Phys. 31 (1959) 646. 